\title{
INVERSE KINEMATICS OF A SERIAL-PARALLEL ROBOT USED IN HOT FORGING PROCESS
}

\author{
Chu Anh My \\ Le Quy Don Technical University, Hanoi, Vietnam \\ E-mail: mychuanh@yahoo.com \\ Received March 12, 2015
}

\begin{abstract}
In hot extrusion forging process, the use of robot arm for transferring heavy billets can reduce downtime, improve productivity, reduce worker fatigue and optimize the use of energy and manpower. To increase the stiffness of the robot and force the endeffector move in directions parallel with the ground surface, two parallel links are added to a standard serial manipulator. This modification of the structure could make it a bit of challenge in the system modelling and controlling. This paper addresses the inverse kinematics analysis that is the central issue for developing autonomous control modes of the robot application.
\end{abstract}

Keywords: Serial-paralell robot, inverse kinematics, hot extrusion forging.

\section{INTRODUCTION}

Forging is a manufacturing process involving the shaping of metal using localized compressive forces. Forging is often classified according to the temperature at which it is performed: "cold", "warm", or "hot" forging. Consider a hot forging process layout in Fig. 1.

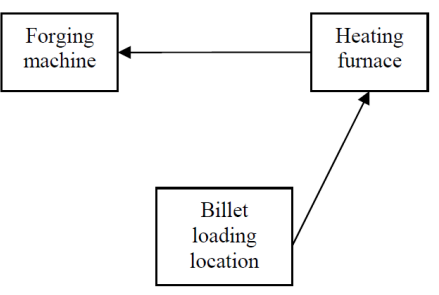

Fig. 1. Layout of forging station

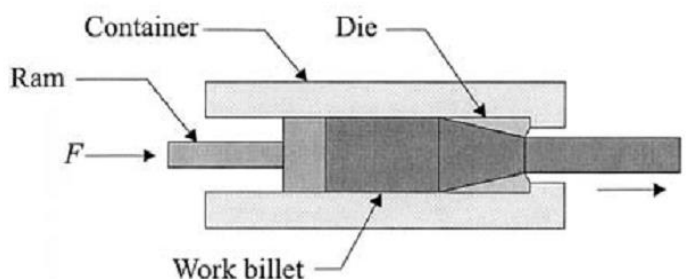

Fig. 2. Forging process 
A steel billet weighted about $60 \mathrm{~kg}$ is heated up to $1100^{\circ} \mathrm{C}$ in the heating furnace, and it is then transferred to the forging machine. The forging machine is a hydraulic press system used for the extrusion process. After the billet located in a die fixed in the machine, it is pressed under the force $F$ by the hydraulic press equipment, to produce the forging part (see Fig. 2).

Since billets are hot and heavy, two workers are required to carry and transfer billets one by one for the process. This causes instability, worker fatigue and downtime in the manufacturing. To overcome these disadvantages, an industrial robotics system shown in Fig. 3 is designed to support the workers.

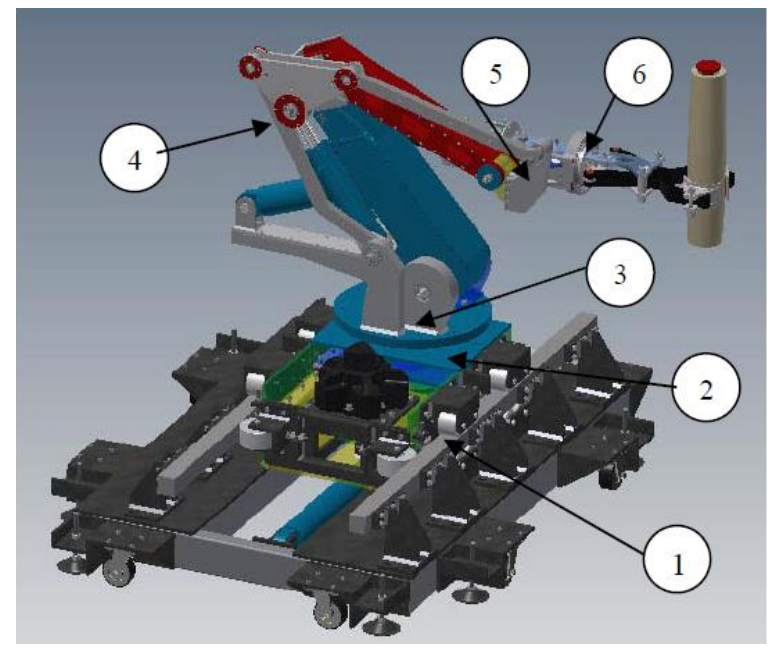

Fig. 3. Robot design for hot forging process

The robot consists of 6 joints, 5 links and one gripper (the end-effector); and all system is actuated by hydraulic cylinders. The first joint connecting the manipulator with the chassis is a translation joint; all the other joints are prismatic. The links connecting joint 3 and 4 , and connecting joint 4 and 5 are parallel mechanisms. This structural modification accompanied with the hydraulic actuators aim to reinforce the stiffness of the system so that the robot is capable of carrying the heavy billet. In addition, the orientation of the end-effector is restricted as desired; the end-link moves in only directions parallel with the ground surface. This advantage could help to reduce the complexity of the control process. Though the hybrid design takes full advantages of flexibility of the serial links and makes full use of the reinforced stiffness of the added parallel mechanisms, it possesses complexity in modelling and controlling.

For controlling the robot, the kinematical modelling and analysis play a central role that needs to be considered specifically. The kinematic of general manipulator has been the fundamental problem [1], in which the serial chain of links is considered as the standard kinematical model. Either Danavit-Hartenberg method or Craig method is usually used for the modelling. Further studies on the manipulator kinematic could be found in the literature such as the kinematics for the redundancy structures [2], the kinematic of 
the parallel robot [3,4], kinematic performance analysis [5], kinematics of manipulator mounted on a mobile plateform [6], and the kinematics design of manipulator [7]. However, a few researcher interests in modeling and analyzing the hybrid serial - parallel robot structures.

This paper addresses the modelling of which the kinematical constraint of the parallel links is taken into account. A numerical solution to the inverse kinematics problem is proposed. Finally, given the trajectory of the end-effector, the time history of joint variables are determined that can be used for the controlling process.

\section{KINEMATICS MODELLING}

In Fig. 4 , we define $d_{1}, q_{2}, q_{3}, q_{4}, q_{5}$ and $q_{6}$ are joint variables which determine the configuration of the mechanical system. We denote $\mathbf{q}=\left[\begin{array}{llllll}d_{1} & q_{2} & q_{3} & q_{4} & q_{5} & q_{6}\end{array}\right]^{T}$. Due to the parallel motion of the two parallel links, the relationship between $q_{3}, q_{4}$ and $q_{5}$ can be illustrated in the following Fig. 5 .

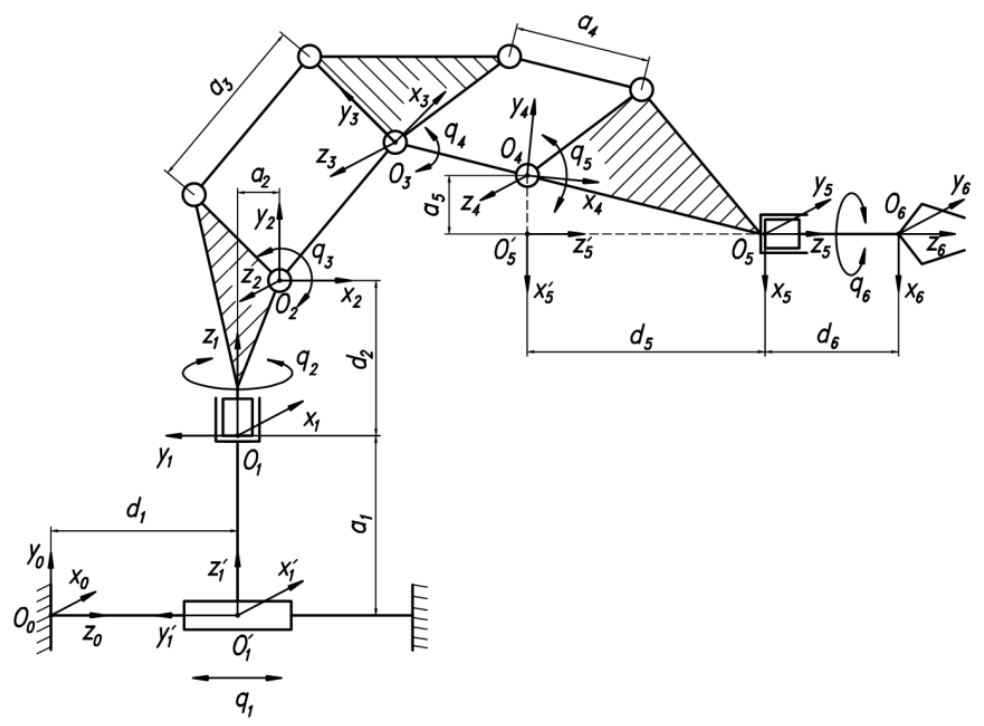

Fig. 4. Kinematical model of serial parallel robot

As shown in the Fig. 5, the following constrain can be write

$$
q_{5}=-q_{3}-q_{4}-\frac{\pi}{2}
$$

Following the Denavit-Hartenberg technique, all joint coordinate systems are denoted as shown in Fig. 4. Also, in Tab. 1, kinematical and geometrical parameters with respect to all links are illustrated. Notice that link number $1^{\prime}$ and $5^{\prime}$ are added to make it easier in writing homogeneous transformation matrixes in the form of the DenavitHartenberg's formulation. $O_{0} \equiv\left(O_{0} x_{0} y_{0} z_{0}\right)$ is denoted as the reference frame. In the 


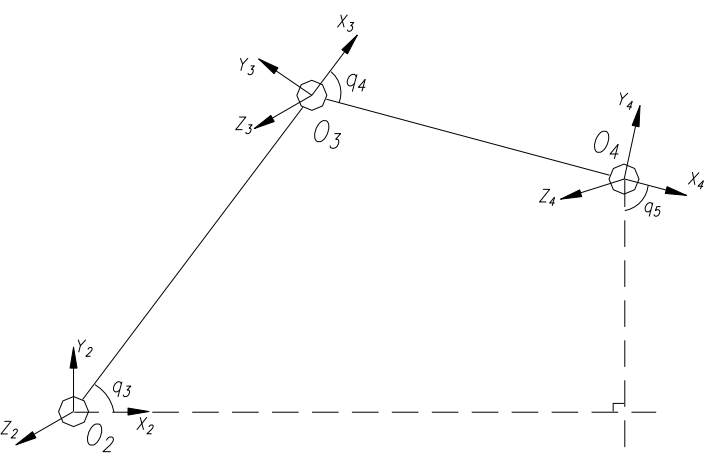

Fig. 5. Geometrical relationship between $q_{3}, q_{4}$ and $q_{5}$

Table 1. Kinematical and geometrical parameters of links

\begin{tabular}{|c|c|c|c|c|}
\hline Link & $\theta_{i}$ & $d_{i}$ & $a_{i}$ & $\alpha_{i}$ \\
\hline $1^{\prime}$ & 0 & $d_{1}$ & 0 & 0 \\
\hline $1^{\prime \prime}$ & 0 & 0 & 0 & $-\pi / 2$ \\
\hline 1 & 0 & $a_{1}$ & 0 & 0 \\
\hline 2 & $q_{2}$ & $d_{2}$ & $a_{2}$ & $\pi / 2$ \\
\hline 3 & $q_{3}$ & 0 & $a_{3}$ & 0 \\
\hline 4 & $q_{4}$ & 0 & $a_{4}$ & 0 \\
\hline $5^{\prime}$ & $q_{5}$ & 0 & $a_{5}$ & $-\pi / 2$ \\
\hline 5 & 0 & $d_{5}$ & 0 & 0 \\
\hline 6 & $q_{6}$ & $d_{6}$ & 0 & 0 \\
\hline
\end{tabular}

same way, the following notations $\mathrm{O}_{1}, \mathrm{O}_{2}, \ldots, \mathrm{O}_{6}$ are the link frames, correspondingly. The frames $\mathrm{O}_{2}^{\prime}, \mathrm{O}_{3}^{\prime}, \mathrm{O}_{4}^{\prime}$ are added for the refencing purpose.

The homogeneous transformation matrix of the end-effector with respect to the reference frame can be denoted as

$$
\mathbf{H}_{0 E}=\left[\begin{array}{cc}
\mathbf{A}_{0 E} & \mathbf{r}_{E} \\
0 & 1
\end{array}\right]
$$

where $\mathbf{r}_{E}=\left[\begin{array}{ccc}x_{E} & y_{E} & z_{E}\end{array}\right]^{T}$ is the position vector of the end-effector; $\mathbf{A}_{0 E}$ is the rotation matrix of the end-effector with respect to the reference frame.

The position and the orientation of the end-effector can be determined via the following relationship

$$
\mathbf{H}_{0 E}=\mathbf{H}_{01^{\prime}}\left(d_{1}\right) \mathbf{H}_{1^{\prime} 1} \mathbf{H}_{12}\left(q_{2}\right) \mathbf{H}_{23}\left(q_{3}\right) \mathbf{H}_{34}\left(q_{4}\right) \mathbf{H}_{45^{\prime}}\left(q_{5}\right) \mathbf{H}_{5^{\prime} 5} \mathbf{H}_{56}\left(q_{6}\right),
$$

where $\mathbf{H}_{j i}$ is the homogeneous transformation matrix defined for the homogeneous motion of the frame indexed $i$ with respect to the previous frame indexed $j$. 


$$
\mathbf{H}_{j i}=\left[\begin{array}{cccc}
\cos \theta_{i} & -\sin \theta_{i} \cos \alpha_{i} & \sin \theta_{i} \sin \alpha_{i} & a_{i} \cos \theta_{i} \\
\sin \theta_{i} & \cos \theta_{i} \cos \alpha_{i} & -\cos \theta_{i} \cos \alpha_{i} & a_{i} \sin \theta_{i} \\
0 & \sin \alpha_{i} & \cos \alpha_{i} & d_{i} \\
0 & 0 & 0 & 1
\end{array}\right] .
$$

Substituting all the matrixes $\mathbf{H}_{j i}$ yielded in accordance with Tab. 1 into Eq. (3) obtains

$$
\left[\begin{array}{cc}
\mathbf{A}_{0 E} & \mathbf{r}_{E} \\
0 & 1
\end{array}\right]=\left[\begin{array}{cc}
\mathbf{A}_{06}(\mathbf{q}) & \mathbf{r}_{06}(\mathbf{q}) \\
0 & 1
\end{array}\right]
$$

Eq. (5) and constraint Eq. (1) describe the forward relationship of the robot. Given a value of $\mathbf{q}$, the position and the orientation of the end-effector can be calculated analytically.

If we denote $\mathbf{f}(\mathbf{q})=\mathbf{r}_{06}(\mathbf{q})$ as function vector of $\mathbf{q}$, for the position forward kinematics, the following equation can be yielded

$$
\mathbf{r}_{E}=\mathbf{f}(\mathbf{q}) .
$$

\section{INVERSE KINEMATICS}

To control the robot operating as desired, the inverse kinematics computation must be taken into account. Generally, given the path of the end-effector, all the histories of the joint variables must be calculated as

$$
\mathbf{q}=\mathbf{f}^{-1}\left(\mathbf{r}_{E}\right) .
$$

For the considered robot, the orientation of the end-effector is regardless because of the feature of the parallel structure mentioned previously. In addition, as discussed before, the robot transfers the billet for forging process in discontinuous sequence. Therefore, the continuity of the tool path is not required. The path of the point $\mathbf{r}_{E}$ of the endeffector is illustrated in the following Fig. 6.

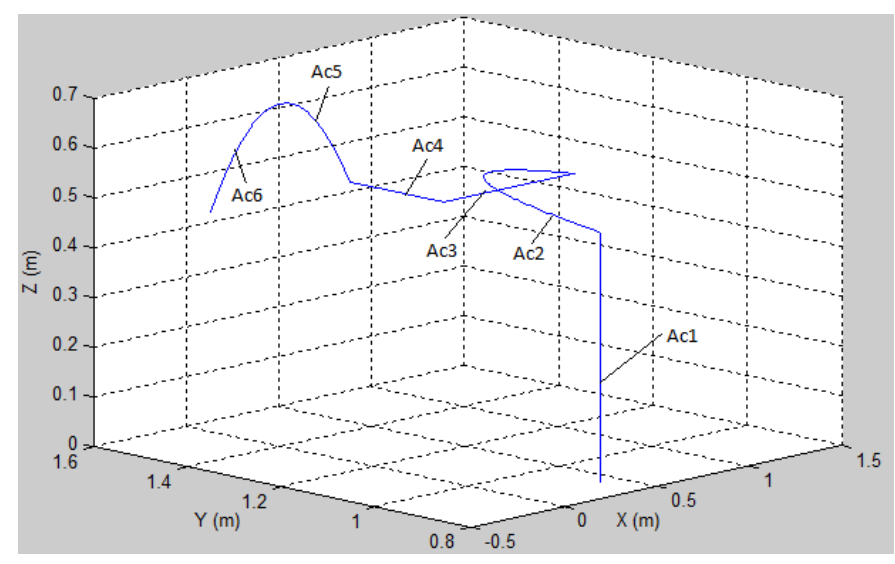

Fig. 6. Required path of the end-effector 
As seen in Fig. 6, the path can be separated into 6 arcs (Ac1,.., Ac6) representing 6 periods of time that the robot fulfills the required task. To simplify the formulation and implementation of the inverse kinematics, Eq. (7) is solved according to the separated 6 periods of time as follows.

Period 1. The robot moves in $z_{0}$ direction only to locate the end-effector and gets ready for picking up the billet

The geometrical parameters are given as: $d_{1}=0.5 \mathrm{~m}, a_{1}=0.11 \mathrm{~m}, d_{2}=0.25 \mathrm{~m}$, $a_{2}=0.1 \mathrm{~m}, a_{3}=0.73 \mathrm{~m}, a_{4}=0.63 \mathrm{~m}, a_{5}=0.18 \mathrm{~m}, d_{5}=0.03 \mathrm{~m}, d_{6}=0.43 \mathrm{~m}$.

The initial values of joint variables are given as follows: $d_{1}=0, q_{2}=0, q_{3}=$ $\frac{\pi}{3}, q_{4}=\frac{\pi}{6}, q_{6}=0$. The end-effector moves so that $x_{E}=0.4, y_{E}=0.88$ and $z_{E}=0.5 t$, where $t \in \Re$ is a parameter and $t \in[0,1]$.

In this period, only $d_{1}$ varies. Finally we obtain $d_{1}=0.5 t$.

Period 2. The robot transfers the billet to the heating furnace

In this period of time, $\mathbf{r}_{E}$ can be represented as

$$
\left\{\begin{array}{l}
x_{E}(t)=-0.124 t^{2}+0.8 t+0.4 \\
y_{E}(t)=-0.92 t^{2}+1.24 t+0.88 \\
z_{E}(t)=0.5
\end{array}\right.
$$

Solving Eq. (7) yields $d_{1}=0.5, q_{2}=0, q_{6}=0$, and $q_{3}, q_{4}$ and $q_{5}$ vary as shown in Fig. 7 .
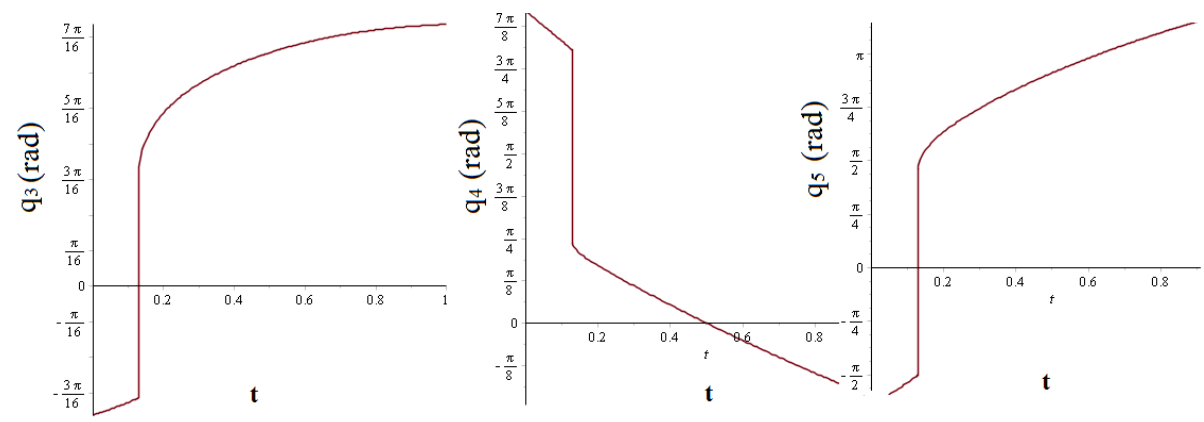

Fig. 7. Values of $q_{3}, q_{4}$ and $q_{5}$ for the period 2

Period 3. The robot picks up the billet heated and moves it outside the furnace

In this period of time, $\mathbf{r}_{E}$ can be represented as

$$
\left\{\begin{array}{l}
x_{E}=1.324-0.95 t \\
y_{E}=1.2 \\
z_{E}=0.5
\end{array}\right.
$$

Solving Eq. (7) yields $d_{1}=0.5, q_{2}=0, q_{6}=0$, and $q_{3}, q_{4}$ and $q_{5}$ as shown in Fig. 8 .

Period 4 . The robot moves the billet up and gets ready to turn transferring the billet to the forging machine 

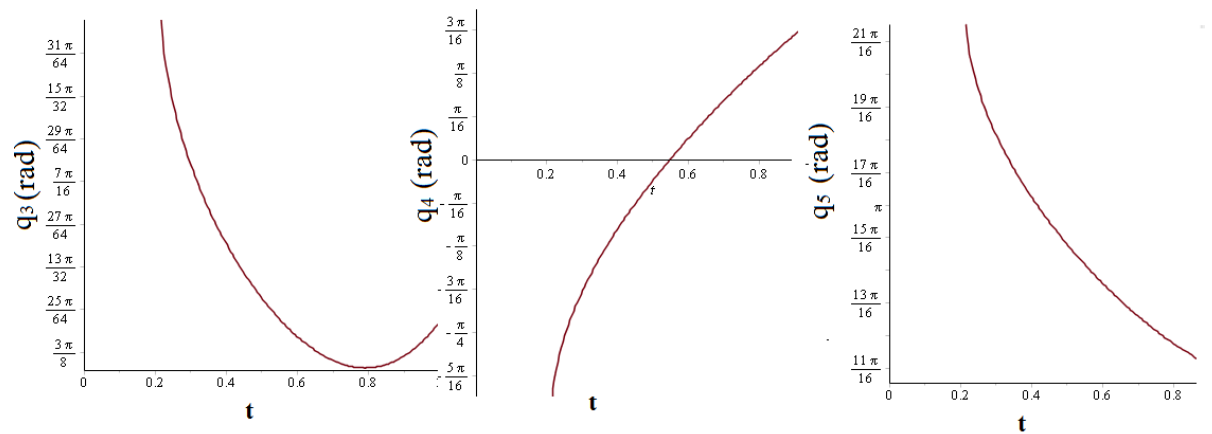

Fig. 8. Values of $q_{3}, q_{4}$ and $q_{5}$ for the period 3

In this period of time, $\mathbf{r}_{E}$ can be represented as

$$
\left\{\begin{array}{l}
x_{E}=0.374 \\
y_{E}=0.2 t+1.2 \\
z_{E}=0.5
\end{array}\right.
$$

Solving Eq. (7) yields $d_{1}=0.5, q_{2}=0, q_{6}=0$, and $q_{3}, q_{4}$ and $q_{5}$ as shown in Fig. 9 .
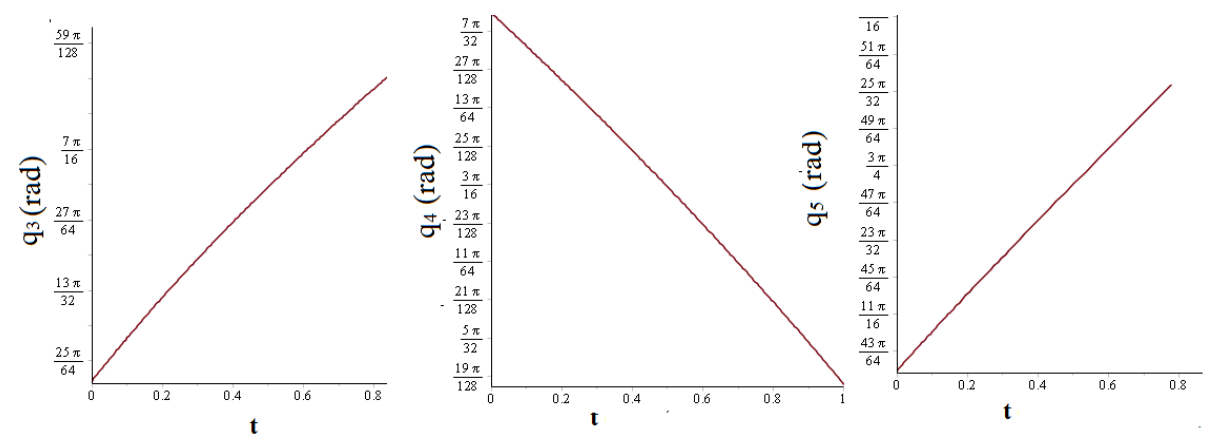

Fig. 9. Values of $q_{3}, q_{4}$ and $q_{5}$ for the period 4

Period 5. The robot turns $180^{\circ}$ moving the billet to the forging machine. After that the end-effector turns $90^{\circ}$

For this period, only $q_{2}$ varies from 0 to $180^{\circ}$. After that $q_{6}$ varies form 0 to $90^{\circ}$. All other joint variables keep their values as calculated in the last period.

Period 6. The robot reaches to the position ready to release the billet on the forging machine

In this period of time, $\mathbf{r}_{E}$ can be represented as

$$
\left\{\begin{array}{l}
x_{E}(t)=0.576 t^{2}-1.452 t-0.374 \\
y_{E}(t)=0.12 t^{2}-0.4 t+1.4 \\
z_{E}(t)=0.5
\end{array}\right.
$$

Solving Eq. (7) yields $d_{1}=0.5, q_{2}=\pi, q_{6}=\frac{\pi}{2}$, and $q_{3}, q_{4}$ and $q_{5}$ as shown in Fig. 10. 


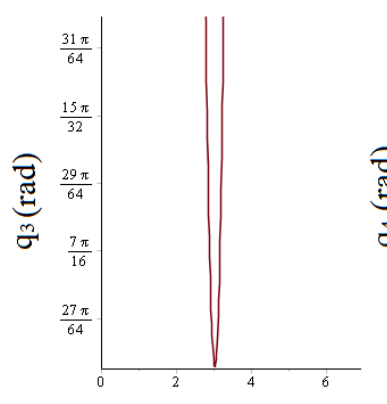

$10^{-1} \mathrm{t}$

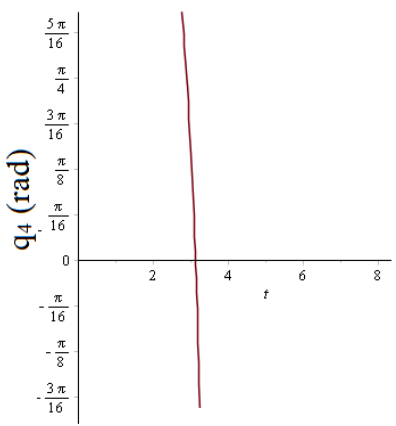

$10^{-1} \mathrm{t}$

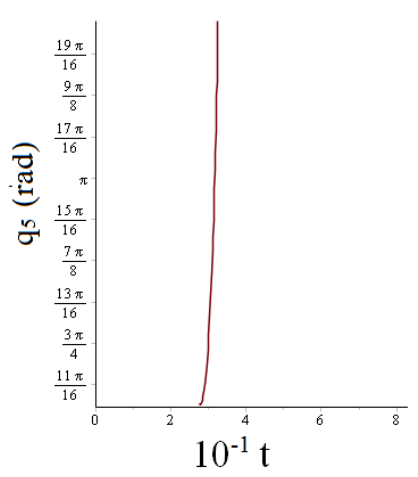

Fig. 10. Values of $q_{3}, q_{4}$ and $q_{5}$ for the period 6

For 6 given segments of the required path, the solution to the inverse position kinematics is found that is applicable to control the joint actuators. The values of the joint variables change in feasible ranges.

\section{CONCLUSION}

The kinematical model of the serial-parallel robot is formulated. The kinematics equation of the considering robot must satisfy the constraint equation because of the motion constrained of the parallel links. This is different from the typical serial robot.

The end-effector moves always parallel with the ground surface that could simplify the control procedure. Moreover, this is to make sure that the griper holds the billet in fixed posture during the transferring time period.

The numerical results of the inverse equations are suitable in value and the curves show its proper change in shape and magnitude.

\section{REFERENCES}

[1] N. V. Khang and C. A. My. Industrial robot basics. Vietnam Education Publishing House, (2011). (in Vietnamese).

[2] J. Wang, Y. Li, and X. Zhao. Inverse kinematics and control of a 7-DOF redundant manipulator based on the closed-loop algorithm. International Journal of Advanced Robotic Systems, 7, (4), (2010), pp. 1-9.

[3] Y. Li and Q. Xu. Kinematic analysis of a 3-PRS parallel manipulator. Robotics and ComputerIntegrated Manufacturing, 23, (4), (2007), pp. 395-408.

[4] J.-P. Merlet. Parallel robots. London Kluwer Academic Publishers, (2012).

[5] K. Balaji, K. Brahma Ralu, and M. Surya Narayana. Kinematic performance analysis of 4-link planar serial manipulator. International Journal of Research in Engineering and Technology, 3, (11), (2014), pp. 179-183.

[6] C. A. My. Mechanical design and dynamics modelling of RoPC robot. In Proceedings of IFToMM International Symposium on Robotics and Mechatronics, (2009).

[7] M. Ceccarelli and E. Ottaviano. Kinematic Design of Manipulators. INTECH Open Access Publisher, (2008). 